Editorial

\title{
Acknowledgement to Reviewers of Journal of Low Power Electronics and Applications in 2017
}

Journal of Low Power Electronics and Applications Editorial Office MDPI AG, St. Alban-Anlage 66, 4052 Basel, Switzerland

Published: 1 January 2018

Peer review is an essential part in the publication process, ensuring that Journal of Low Power Electronics and Applications maintains high quality standards for its published papers. In 2017, a total of 31 papers were published in the journal. Thanks to the cooperation of our reviewers, the median time to first decision was 28 days and the median time to publication was 66 days. The editors would like to express their sincere gratitude to the following reviewers for their time and dedication in 2017:

Abbas, Zia

Agarwal, Harshit

Agyeman, Michael O.

Aktaruzzaman, $\mathrm{Md}$

Alander, Jarmo

Anders, Jens

Angione, Maria Daniela

Assadian, Francis

Ax, Johannes

Bai, Yu

Beckett, Paul

Bernardi, Giulio

Beyl, Tim

Bi, Bill

Bosio, Alberto

Chang, Young

Chauhan, Munish

Chen, Changlin

Chen, Kun-Chih

Chen, Roger Yubtzuan

Chen, Yie-Tone

Chew, Zheng Jun

Choi, Jaehyuk

Cirlugea, Mihaela

Corsonello, Pasquale

Costa, Nuno

Crupi, Felice

Díaz, Antonio F.
Echeto, Francesc Moll

Eghbal, Ashkan

Ewetz, Rickard

Flandre, Denis

Fraga-Lamas, Paula

Fularz, Michal

Ganguly, Amlan

Genovese, Angelo

Ghibaudo, G.

Giorgetti, Andrea

Graham, David W.

Grasso, Alfio Dario

Guo, Xinfei

Guo, Zhaoqin

Gupta, Sumeet Kumar

Ho, Weng-Geng

Horng, Jiun-Wei

$\mathrm{Hu}$, Wei

Huang, Shu-Chuan

Imtiaz, Syed Anas

Isern, Eugeni

Jalalifar, Majid

Jiao, Hailong

Juang, Tso-Bing

Jurišić, Dražen

Kandasamy, Nandha Kumar

Karlin, Ian

Kassanos, Panagiotis 
Kaufmann, Steffen

Khushwaha, Chandrabhan Singh

Kiayani, Adnan

Kim, Jusung

Kornaros, George

Kotb, Jabeur

Kryjak, Tomasz

Kshirsagar, Chaitanya

Kuo, Chien-Nan

Kushwaha, Pragya

Labati, Ruggero Donida

Lapray, Pierre-Jean

Li, Jian-yu

Li, Zhaolin

Libertino, Sebania

Loveless, T Daniel

Lu, Yan

Mahapatra, Rabi N.

Makosiej, Adam

Margetts, Rebecca

Marzband, Mousa

Miller, David Michael

Min, Mart

Mineo, Andrea

Monteleone, Salvatore

Moreira, Matheus Trevisan

Motaman, Seyedhamidreza

Ngwompo, Roger

Padovani, Andrea

Palesi, Maurizio

Pan, Shangzhi

Panagiotakis, Spyros

Park, Jongsun

Patel, Sarosh

Pehl, Michael

Perez-Nicoli, Pablo

Qadri, Muhammad Yasir
Ragulskis, Minvydas

Razaque, Abdul

Record, Paul

Reverter, Ferran

Richelli, Anna

Rinkeviciene, Roma

Rizzolo, Serena

Rossi, Davide

Salman, Emre

Schmidt, Adam

Sikora, Andrzej

Silva-Martinez, Jose

Singer, Sigmond

Slesazeck, Stefan

Smith, Scott C.

Song, Taigon

Sotner, Roman

Thern, Marcus

Tognetti, Alessandro

Tribioli, Laura

Tsai, Zuo-Min

Valkama, Mikko

Velez, Gorka

Venkatesan, Rangharajan

Vliegen, Jo

Wen, Chih-Yu

Whatmough, Paul

Wiest, Joachim

Wu, Guoying

$\mathrm{Wu}$, Heng

$\mathrm{Wu}$, Wen-Jong

Xiang, Dong

Yang, Hao-I

Yang, Shufan

Yao, Lina

Zhang, Tong

Zhou, Jun

(C) 2018 by the authors. Submitted for possible open access publication under the terms and conditions of the Creative Commons Attribution (CC-BY) license (http://creativecommons.org/licenses/by/4.0/). 